\title{
Auxotrophic Actinobacillus pleurpneumoniae grows in multispecies biofilms without the need for nicotinamide-adenine dinucleotide (NAD) supplementation
}

Abraham Loera-Muro ${ }^{1}$, Mario Jacques², Francisco J. Avelar-González¹, Josée Labrie², Yannick D. N. Tremblay², Ricardo Oropeza-Navarro ${ }^{3}$ and Alma L. Guerrero-Barrera ${ }^{1,4^{*}}$

\begin{abstract}
Background: Actinobacillus pleuropneumoniae is the etiologic agent of porcine contagious pleuropneumonia, which causes important worldwide economic losses in the swine industry. Several respiratory tract infections are associated with biofilm formation, and A. pleuropneumoniae has the ability to form biofilms in vitro. Biofilms are structured communities of bacterial cells enclosed in a self-produced polymer matrix that are attached to an abiotic or biotic surface. Virtually all bacteria can grow as a biofilm, and multi-species biofilms are the most common form of microbial growth in nature. The goal of this study was to determine the ability of A. pleuropneumoniae to form multi-species biofilms with other bacteria frequently founded in pig farms, in the absence of pyridine compounds (nicotinamide mononucleotide [NMN], nicotinamide riboside [NR] or nicotinamide adenine dinucleotide [NAD]) that are essential for the growth of A. pleuropneumoniae.
\end{abstract}

Results: For the biofilm assay, strain 719, a field isolate of A. pleuropneumoniae serovar 1, was mixed with swine isolates of Streptococcus suis, Bordetella bronchiseptica, Pasteurella multocida, Staphylococcus aureus or Escherichia coli, and deposited in 96-well microtiter plates. Based on the CFU results, A. pleuropneumoniae was able to grow with every species tested in the absence of pyridine compounds in the culture media. Interestingly, A. pleuropneumoniae was also able to form strong biofilms when mixed with S. suis, B. bronchiseptica or S. aureus. In the presence of $E$. coli, A. pleuropneumoniae only formed a weak biofilm. The live and dead populations, and the matrix composition of multi-species biofilms were also characterized using fluorescent markers and enzyme treatments. The results indicated that poly- $\mathrm{N}$-acetyl-glucosamine remains the primary component responsible for the biofilm structure.

Conclusions: In conclusion, A. pleuropneumoniae apparently is able to satisfy the requirement of pyridine compounds through of other swine pathogens by cross-feeding, which enables A. pleuropneumoniae to grow and form multi-species biofilms.

Keywords: Biofilms, Actinobacillus pleuropneumoniae, Streptococcus suis, Bordetella bronchiseptica, Pasteurella multocida, Staphylococcus aureus, Escherichia coli, Pyridine compounds

\footnotetext{
* Correspondence: alguerre@correo.uaa.mx

${ }^{1}$ Centro de Ciencias Básicas, Universidad Autónoma de Aguascalientes, Aguascalientes, Ags., Mexico20131

${ }^{4}$ Laboratorio de Biología Celular y Tisular, Departamento de Morfología,

Centro de Ciencias Básicas, Universidad Autónoma de Aguascalientes,

Aguascalientes, Ags., Mexico20131

Full list of author information is available at the end of the article
}

(c) 2016 The Author(s). Open Access This article is distributed under the terms of the Creative Commons Attribution 4.0 International License (http://creativecommons.org/licenses/by/4.0/), which permits unrestricted use, distribution, and reproduction in any medium, provided you give appropriate credit to the original author(s) and the source, provide a link to the Creative Commons license, and indicate if changes were made. The Creative Commons Public Domain Dedication waiver (http://creativecommons.org/publicdomain/zero/1.0/) applies to the data made available in this article, unless otherwise stated. 


\section{Background}

Actinobacillus pleuropneumoniae is the etiologic agent of porcine contagious pleuropneumonia, an infectious disease of swine, which causes important economic losses in the pig industry worldwide [1-7]. A. pleuropneumoniae is a Gram-negative bacteria belonging to the Pasteurellaceae family [8-11]. Two biotypes have been described based on their dependence of nicotinamide adenine dinucleotide (NAD) and fifteen serovars are recognized $[8,12]$.

Many virulence factors have been reported in $A$. pleuropneumoniae $[5,6,8,13,14]$, which include adhesion structures, such as type IV pilus [5, 15, 16], Flp pilus [3], and autotransporter adhesins [5], and biofilms formation $[3,5,17]$. Other swine pathogens, including Bordetella bronchiseptica, Escherichia coli, Haemophilus parasuis, Salmonella typhymurium, Staphylococcus aureus, and Streptococcus suis, can also form biofilms [9, 18].

Biofilms are structured communities of microorganisms, enclosed in a self-produced extracellular polymer matrix, attached to biological or non-biological surfaces [19-21]. Biofilms can be found in every ecosystem including natural, engineered and pathogenic settings. Growth as a biofilm is considered to be a protective mode that allows for survival in hostile environments [9]. Multi-species biofilms is likely the most prominent lifestyle of microorganisms outside the laboratory and the species composition of these biofilms will vary and be dependent on the environment [20, 22-25]. In multispecies biofilms, microorganisms will communicate, compete or cooperate to improve and ensure their survival and propagation [22, 24].

The goal of this study was to determine the ability of the swine respiratory pathogen $A$. pleuropneumoniae biotype 1 , serovar 1 to form multi-species biofilms with other swine bacterial pathogens (S. suis, B. bronchiseptica, P. multocida, S. aureus or E. coli) that frequently are present in swine farms, in the absence of pyridine compounds (nicotinamide mononucleotide [NMN], nicotinamide riboside [NR] or nicotinamide adenine dinucleotide [NAD]). Pyridine compounds are essential for the growth of $A$. pleuropneumoniae biotype 1 . The live and dead populations, and the matrix composition of multi-species biofilms were also characterized using fluorescent markers and enzyme treatments.

\section{Methods}

\section{Bacterial strains}

Bacterial strains selected for this study were as follows: A. pleuropneumoniae biotype 1/ serovar 1 strain 719, three bacterial species belonging to the porcine respiratory disease complex (PRDC; S. suis serovar 2 strain 735, B. bronchiseptica strain 276, and Pasteurella multocida D strain 1703), a $S$. aureus isolated previously from a healthy pig (strain $154 \mathrm{~N}$ ) and an enterotoxigenic E. coli (ETEC) isolated previously from pig (strain ECL17608). All bacteria were grown on brain heart infusion agar plates (BHI; Oxoid Ltd, Basingstoke, Hampshire, UK) with supplementation of $15 \mu \mathrm{g} / \mathrm{mL}$ NAD for A. pleuropneumoniae and only $\mathrm{BHI}$ for all the other bacteria. Plates were incubated overnight at $37{ }^{\circ} \mathrm{C}$ with $5 \% \mathrm{CO}_{2}$. A colony was transferred into $5 \mathrm{~mL}$ BHI (Oxoid) with $15 \mu \mathrm{g}$ / $\mathrm{mL}$ NAD or without this supplementation, and incubated at $37{ }^{\circ} \mathrm{C}$ overnight with agitation. This overnight culture was used for the biofilm assays. In this study no animal was utilized, because all experiments were done in vitro.

\section{Multi-species biofilm assay}

Multi-species biofilm assays were performed as described previously Labrie et al. [17] for single-species with some modifications (Table 1). Briefly, overnight cultures of A. pleuropneumoniae, S. suis, B. bronchiseptica, P. multocida, E. coli or S. aureus were diluted $1 / 100$ in BHI with and without supplementation of NAD. Volumes of the dilution were aliquoted by triplicate in wells of a sterile 96-well microtiter plate (Costar 3599, Corning, NY, USA) using the following template as an example: $100 \mu \mathrm{L}$ A. pleuropneumoniae in BHI-NAD + $100 \mu \mathrm{L}$ S. suis in BHI-NAD, or $100 \mu \mathrm{L}$ A. pleuropneumoniae in $\mathrm{BHI}+100 \mu \mathrm{L} S$. suis in BHI. The same template was used for the following combination: A. pleuropneumoniae - B. bronchiseptica, A. pleuropneumoniae - $P$. multocida, A. pleuropneumoniae - E. coli, and A. pleuropneumoniae - S. aureus. For the triple-species biofilms, $50 \mu \mathrm{L}$ of each species were aliquoted in the wells. The triple-species combinations were as followed: A. pleuropneumoniae - S. suis - B. bronchiseptica, A. pleuropneumoniae - S. suis - E. coli; and A. pleuropneumoniae - B. bronchiseptica - E. coli. For single species controls, $75 \mu \mathrm{L}$ of a species dilution in BHI-NAD was mixed with $75 \mu \mathrm{L}$ of BHI-NAD, or $75 \mu \mathrm{L}$ of a species dilution in BHI was mixed with $75 \mu \mathrm{L}$ of BHI. Wells containing sterile broth were used as negative controls. Following an incubation of $24 \mathrm{~h}$ at $37^{\circ} \mathrm{C}$, the wells were washed by immersion in water and excess water was removed by inverting plates onto a paper towel. Biofilms were then stained with $0.1 \%(\mathrm{w} / \mathrm{v})$ crystal violet for $2 \mathrm{~min}$, washed once with distilled water, dried at $37{ }^{\circ} \mathrm{C}$ for $30 \mathrm{~min}$, and then $100 \mu \mathrm{L}$ of ethanol (70 \%) were added to the wells. Absorbance was measured at $590 \mathrm{~nm}$ using a spectrophotometer (Powerwave, BioTek Instruments, Winooski, VT, USA).

\section{Colony Forming Unit (CFU) counts}

Colony forming units (CFU) of A. pleuropneumoniae and the other bacteria from biofilms were counted, using selective growth media and colony morphology. Briefly, 
multi-species biofilms were prepared as described above, the biofilms were washed once with sterile MilliQ water $(200 \mu \mathrm{L})$ and the biofilms were detached using micropipette [26]. The samples were then serially diluted in $\mathrm{NaCl}$ (0.85\%). Dilutions were plated on the following media: BHI and BHI-NAD for A. pleuropneumoniae - $B$. bronchiseptica and A. pleuropneumoniae - E. coli, blood agar and blood agar-NAD $(15 \mu \mathrm{g} / \mathrm{mL})$ for A. pleuropneumoniae - S. suis and A. pleuropneumoniae - P. multocida, BHI-NAD-crystal violet $(1 \mu \mathrm{g} / \mathrm{mL})$ and mannitol salt agar for A. pleuropneumoniae - S. aureus (all media from Oxoid). Plates were incubated overnight at $37^{\circ} \mathrm{C}$ with $5 \% \mathrm{CO}_{2}$. The colonies grown on these selective media plates were then identified by colony morphology and counted, allowing for an estimation of the relative bacterial composition of multi-species biofilms.

\section{Confocal laser scanning microscopy (CLSM)}

To determine the composition of the biofilm matrix, multi-species biofilms were prepared as described above and stained with FilmTracer FM 1-43 (Invitrogen, Eugene, OR), FilmTracer LIVEDEAD Biofilm Viability Kit (Invitrogen), Wheat Germ Agglutinin (WGA-Oregon Green 488, Molecular Probes; which binds $N$-acetyl-Dglucosamine [PGA] and $N$-acetylneuraminic acid residues), FilmTracerTM SYPRO ${ }^{\circ}$ Ruby biofilm matrix stain (Molecular Probes; binds proteins) or $\mathrm{BOBO}^{\mathrm{TM}}-3$ iodide (Molecular Probes; label extracellular DNA or eDNA) as prescribed by the manufacturer. After $30 \mathrm{~min}$ incubation at room temperature, the fluorescent marker solution was removed, and the biofilms were washed with water. The wells were then filled with $100 \mu \mathrm{L}$ of water or PBS for WGA-stained biofilms. The stained biofilms were visualized by CLSM (FV1000 IX81; Olympus, Markham, ON, Canada) and images were acquired using Fluoview software (Olympus).

\section{Enzymatic treatments of multi-species biofilms}

Assays were performed in order to determine the stability of biofilms to enzymatic degradation. The enzymatic treatment assays were performed as described previously Tremblay et al. [27]. Biofilms were prepared as described above and $50 \mu \mathrm{L}$ of dispersin B $(100 \mu \mathrm{g} / \mathrm{mL}$ in PBS; Kane Biotech Inc., Winnipeg, MB, Canada), $50 \mu \mathrm{L}$ of DNase I $\left.(500 \mu \mathrm{g} / \mathrm{mL} \text { in } 150 \mathrm{mM} \mathrm{NaCl}, 1 \mathrm{mM} \mathrm{CaCl})_{2}\right)$, or $50 \mu \mathrm{L}$ of

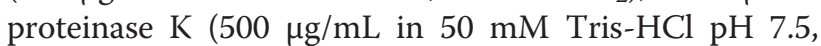
$1 \mathrm{mM} \mathrm{CaCl}_{2}$ ) were added directly to the biofilms. Samples with dispersin $\mathrm{B}$ were incubated for $5 \mathrm{~min}$ at $37{ }^{\circ} \mathrm{C}$ and samples with proteins $\mathrm{K}$ or DNase I were incubated for $1 \mathrm{~h}$ at $37{ }^{\circ} \mathrm{C}$. Control wells were treated with $50 \mu \mathrm{L}$ of the buffer without the enzyme. Biofilms were washed and then stained with crystal violet and the absorbance was measured at $590 \mathrm{~nm}$.

\section{Fluorescent in situ hybridization (FISH)}

In order to confirm the presence of A. pleuropneumoniae and determine its distribution and localization in the biofilms, FISH was performed as described LoeraMuro et al. [7] and Jensen et al. [28] with modifications. Single-species biofilms of $A$. pleuropneumoniae strain 719 was used as a positive control. Single-species biofilms of S. suis strain 735 was used as a probe-specificity control, and was grown as described by Wu et al. [18].

Biofilms were formed on glass slides with flexiPERM (eight wells; Sarstedt, Nümbrecht, Germany), by placing a glass slide in a Petri dish. A volume $(300 \mu \mathrm{L})$ of dilution 1/ 100 of A. pleuropneumoniae or S. suis culture were added to the wells for single-species biofilms and $150 \mu \mathrm{L}$ dilution 1/100 of $A$. pleuropneumoniae and $150 \mu \mathrm{L}$ of $S$. suis or $B$. bronchiseptica culture were added to wells for dualspecies biofilms. The biofilms were incubated for $24 \mathrm{~h}$ at $37{ }^{\circ} \mathrm{C}$ with $5 \% \mathrm{CO}_{2}$. The slides were then air-dried $(1 \mathrm{~h}$ at $37^{\circ} \mathrm{C}$ ) and gently flamed. The biofilms were dehydrated in $100 \%$ alcohol for $30 \mathrm{~min}$ before hybridization. The hybridization was carried out at $45{ }^{\circ} \mathrm{C}$ with $40 \mathrm{~mL}$ of hybridization buffer (100 mM Tris- $\mathrm{HCl}$ [pH 7.2], $0.9 \mathrm{M}$ $\mathrm{NaCl}, 0.1 \%$ sodium dodecyl sulfate) and $200 \mathrm{ng}$ of each probe (APXIVAN-Forward [GGG GAC GTA ACT CGG TGA TT] and APXIVAN-Reverse [GCT CAC CAA CGT TTG CTC] labelled with 633 Alexa Fluor) for $16 \mathrm{~h}$ in a slide rack in the dark. The samples were then washed one time in prewarmed $\left(45^{\circ} \mathrm{C}\right)$ hybridization buffer for $15 \mathrm{~min}$ and subsequently one time in prewarmed $\left(45^{\circ} \mathrm{C}\right)$ washing solution (100 mM Tris-HCl [pH 7.2], 0.9 M NaCl). Samples were then washed with water for $5 \mathrm{~min}$. To localize every bacterial cells, biofilms were also stained with FilmTracerTM FM 1-43 fluorescent marker (Molecular Probes) according to manufacturer's instructions after the hybridization step. To stain with FM 1-43, biofilms were incubated for $30 \mathrm{~min}$ at room temperature in the dark and then washed with water for $10 \mathrm{~min}$. The samples were then covered with ProLong Gold antifade reagent (Invitrogen). The labeled bacteria were visualized using a CLSM (FV1000 IX81; Olympus) and images were acquired using Fluoview software (Olympus).

\section{Statistical analysis}

All the statistical significance $(p$ value $<0.05)$ analyses of differences in biofilm phenotypes (mean optical density values) were determined by a paired, one-tailed $t$-test using GraphPad Prism version 4.0 (GraphPad Software, San Diego, CA, USA).

\section{Results}

Multi-species biofilms formation with NAD supplementation

A. pleuropneumoniae serovar 1 strain 719, a field isolate, was used in a 96-well microtiter plate in multi-species 
biofilms assays. Under favorable growth conditions for A. pleuropneumoniae biofilm formation, bacteria belonging to PRCD (S. suis, B. bronchiseptica and P. multocida) did not reduce or inhibited biofilm formation by $A$. pleuropneumoniae (Fig. 1b). By contrast, the presence of E. coli resulted in a decreased biofilm formation (Fig. 1b). In order to confirm that the other species were present in these biofilms, CFU of $A$. pleuropneumoniae and the other bacteria were determined (Fig. $1 \mathrm{~d}-1 \mathrm{f}$ ) and, in all cases, A. pleuropneumoniae and the other bacterial species were able to grow.
Because $E$. coli causes a decrease in the biofilm of $A$. pleuropneumoniae, a third species was added to the biofilm (S. suis or B. bronchiseptica) to determine if this negative effect presented by the $E$. coli dual-species biofilm was counteracted by the addition of a third bacteria species, forming a triple-species biofilm. This was done because in dual-species biofilm with $S$. suis and $B$. bronchiseptica, A. pleuropneumoniae was still able to form a strong biofilm (OD 2.505). However, E. coli still inhibited biofilm formation by $A$. pleuropneumoniae (OD 0.717, Fig. 1c) when a third species was added.

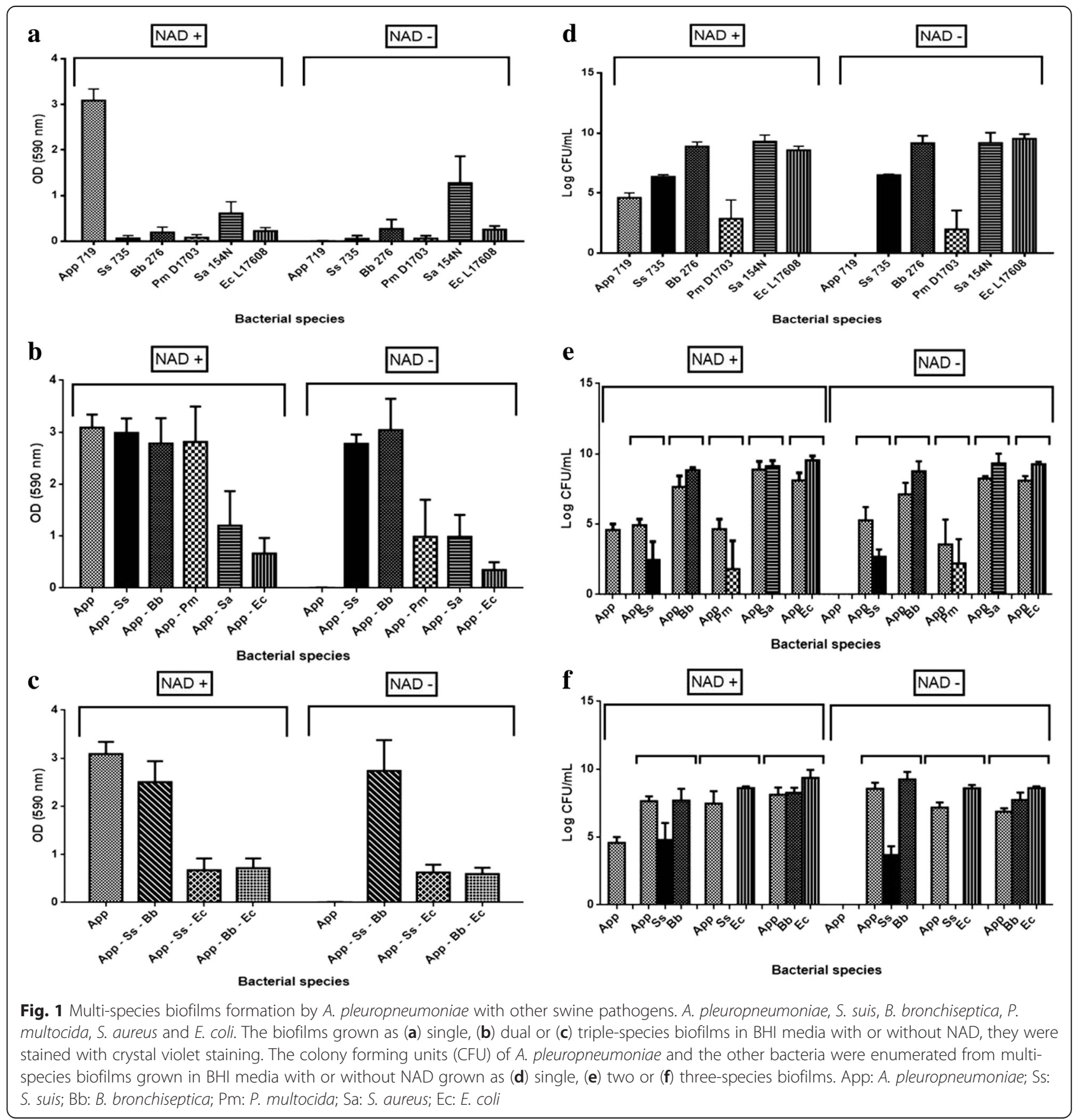


Furthermore, E. coli prevented the growth of S. suis, as determined by CFU counts.

\section{Multi-species biofilms matrix composition with NAD supplementation}

To understand if the addition of a second species had impact on the composition and structure of the extracellular matrix of $A$. pleuropneumoniae biofilms, the biofilms were stained with fluorescent markers or treated with enzymes (Additional file 1:Figure S1). When PGA was labeling, some structural differences were observed. In most dual-species biofilms, PGA distribution appears to be in clusters or filament-like structures, whereas in A. pleuropneumoniae single-species biofilms, PGA distribution was homogeneous. Dispersin B, an enzyme that catalyzes the hydrolysis of linear polymers of $\mathrm{N}$-acetylD-glucosamines, was able to disperse every multi-species biofilms (Fig. 2c). This suggests that PGA was the primary structural component seen in those multi-species biofilms. However, some significant increments in the resistance against the action of this enzyme in some multi-species biofilms were observed. These increments were observed in the two-species biofilms with $S$. aureus (21\%) and E. coli $(24 \%)$, and also in all the threespecies biofilms with E. coli (increased between 20$68 \%$ ) (Fig. 2c).

When eDNA was labeled with $\mathrm{BOBO}^{\mathrm{TM}}-3$ iodide, several differences were observed. With NAD supplementation, increases of eDNA were observed in the twospecies biofilms formed with $B$. bronchiseptica, P. multocida, S. aureus and E. coli; and also in the three-species biofilms with $S$. suis and B. bronchiseptica (Additional file 1: Figure S1). Moreover, proteins not showed significant changes in most two-species biofilms, when they compared with the A. pleuropneumoniae mono-species biofilms. Only in the biofilms of A. pleuropneumoniae $S$. suis, and in all biofilms with E. coli (two and threespecies) the proteins composition were appreciated slightly lower. However, when they were treated with proteinase $\mathrm{K}$ and DNAse $\mathrm{I}$, the biofilms remained attached to the surface indicating that proteins and eDNA do not participate in the integrity of the biofilms (Fig. 2a and $b$ ).

The labeling with FilmTracer FM 1-43, which inserts into the surface membrane on all bacteria, helped to evaluate the morphology of the biofilms. The morphology of two-species biofilms showed low changes, compared with the A. pleuropneumoniae mono-species biofilms. Moreover, in the three-species biofilms formed by A. pleuropneumoniae, S. suis and $B$. bronchiseptica, there was an increase in the occurrence of clusters, showed by fluorescence enhancement (Additional file 1: Figure S1).
When the composition of live/dead cells was evaluated in multispecies biofilms several changes were observed. Alive cells labeled with Syto9 were increased when biofilms of $A$. pleuropneumoniae were performed with $B$. bronchiseptica; with $S$. aureus or with $E$. coli, the same changes were observed also in three species biofilms. These results correlated also with CFU number detected in these multi-species biofilm when they were compared with the A. pleuropneumoniae mono-species biofilms.

Finally, dead cells were labeled with propidium iodide, and two scenarios were founded: the first was an increment of dead bacteria in biofilms of A pleuropneumoniae - B. bronchiseptica, A. pleuropneumoniae - P. multocida and in the three-species A. pleuropneumoniae - B. bronchiseptica - S. suis. The second was the decreased amount of dead bacteria in multi-species biofilms of A. pleuropneumoniae with $S$. aureus and E. coli. In the first three cases, this increment can be related to the amount of eDNA observed, which also increases. In the last cases, also an increment is observed in the amount of eDNA. More studies about the specific compounds present in the extracellular matrix of these biofilms are necessary to understand which changes are produced for the presence the other species in biofilms with A. pleuropneumoniae.

\section{Multi-species biofilms formation without NAD supplementation}

For growth in vitro, A. pleuropneumoniae biotype 1 requires the addition of a pyridine source, which includes NMN, NR or NAD, in the growth media. It is well established that A. pleuropneumoniae can growth without NAD supplementation in presence of $S$. aureus [29]. To test if A. pleuropneumoniae could grow and satisfy the requirements of pyridine compounds through of other swine pathogen species and also form biofilms, the multi-species biofilms assays were also performed in the absence of NAD. Based on OD and CFU results, $A$. pleuropneumoniae was able to grow, form multi-species biofilms and satisfy the requirements of pyridine compounds with every bacterial species tested in this study. A. pleuropneumoniae was able to form strong biofilms with the swine respiratory pathogens $S$. suis (OD 2.762) and B. bronchiseptica (OD 3.042). A. pleuropneumoniae formed a biofilm with the nasal isolate, $S$. aureus that was similar to the one with NAD supplement; however, this dual-species biofilm did not reach the same level as the $A$. pleuropneumoniae single-species biofilm. $A$. pleuropneumoniae - $P$. multocida dual-species biofilms were significant weaker (OD 0.987, $p<0.001$ ) than with NAD supplementation (OD 2.811). As observed before, A. pleuropneumoniae formed a weak biofilm in the presence E. coli (OD 0.349). 

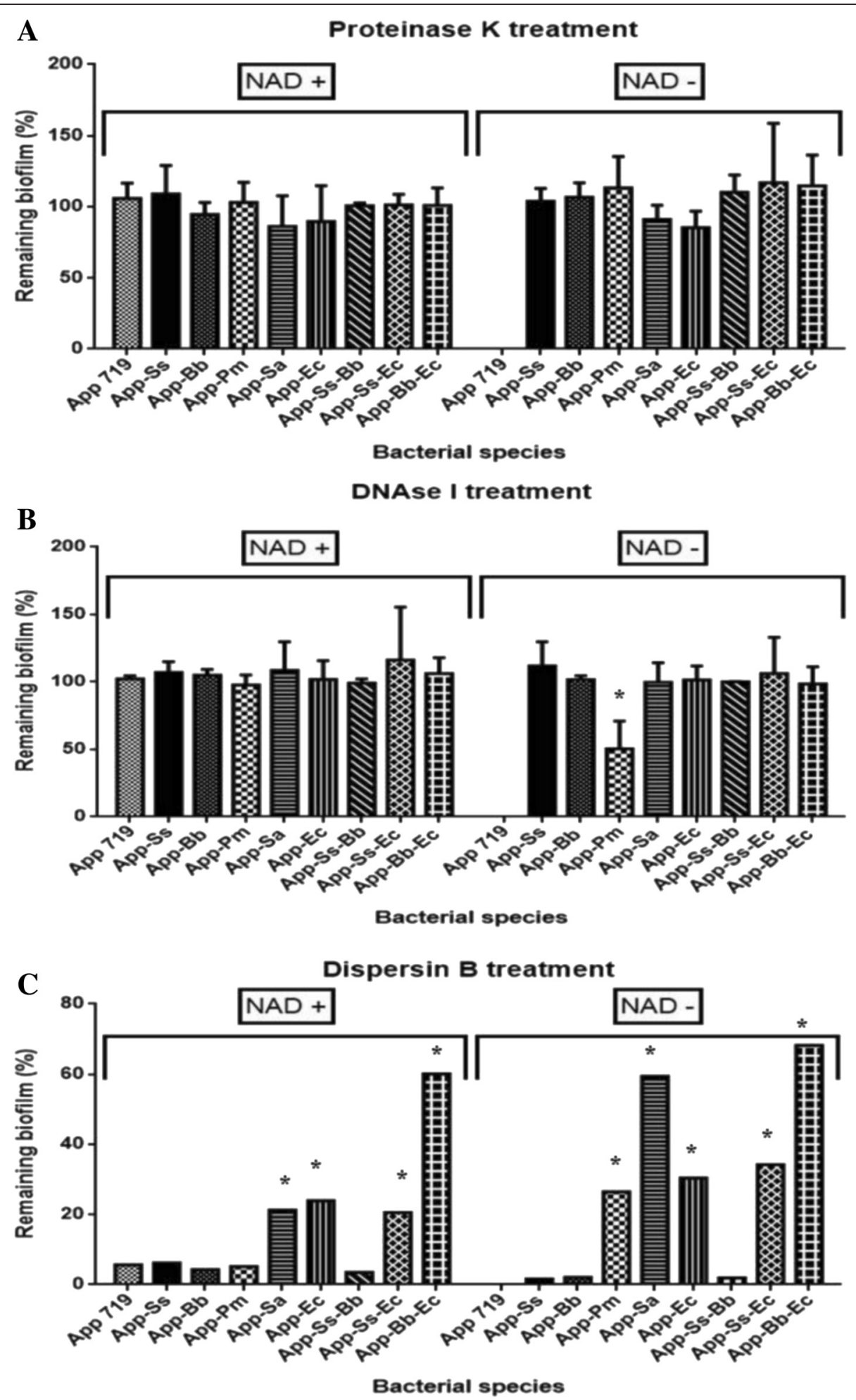

Fig. 2 Effect of enzymatic treatment on multi-species biofilms. Dispersion by (a) proteinase K, (b) DNase I, and (c) dispersin B of multi-species biofilms formed by A. pleuropneumoniae, S. suis, B. bronchiseptica, P. multocida, S. aureus and E. coli grown in BHI media with or without NAD. App: A. pleuropneumoniae; Ss: S. suis; Bb: B. bronchiseptica; Pm: P. multocida; Sa: S. aureus; Ec: E. coli. * $p<0.05$ 
Based on the CFU counts, the number of cells and species ratio did not change in the presence or absence of NAD (Fig. 1e). Thus, the absence of NAD did not have a significant impact species composition of the mix biofilms (Fig. 1e). This suggests that the absence of NAD was not a limiting factor for $A$. pleuropneumoniae growth in the presence of these bacteria species.

The proportions of live/dead bacteria in the multispecies biofilms were also observed with live/dead staining and CLSM. In the presence of P. multocida, E. coli or $S$. aureus, the dead bacteria population decreased when compared to the A. pleuropneumoniae control in BHI-NAD.

Regarding with the triple-species biofilms, again $A$. pleuropneumoniae was able to form strong biofilms with S. suis - B. bronchiseptica (OD 2.737) and weak biofilms when $E$. coli was present (OD 0.594, Fig. 1c). Furthermore, the species ratios were similar to the in the triplespecies biofilms formed with NAD supplementation (Fig. 1f).

\section{Multi-species biofilms matrix composition without NAD supplementation}

The composition of the extracellular matrix was characterized with fluorescent staining or enzymatic digestion (Fig. 2 and 3). Every multi-species biofilms were stained by WGA showing PGA in the biofilm. It was observed as clusters or with filament-like in some biofilms, PGA distribution was not homogenous in the multi-species biofilms. As was described before, all biofilms were sensitive to dispersin B treatment and this support that PGA is present in the biofilm; however, some biofilms were more resistant to the treatment. For example, $59 \%$ and $75 \%$ of the $A$. pleuropneumoniae-S. aureus biofilm and the A. pleuropneumoniae - B. bronchiseptica - E. coli biofilm remained after the treatment. This suggests that other components are important for the integrity of those biofilms.

For eDNA, an increase in the amount of stained eDNA was observed in the $A$. pleuropneumoniae- $B$. bronchiseptica and A. pleuropneumoniae-P. multocida biofilm (Fig. 3). Furthermore, the biofilm of $A$. pleuropneumoniae- $P$. multocida was sensitive to the treatment with DNase I and $50 \%$ of the biofilm remained attached. Every other biofilms were resistant to DNase I treatment. This shows that eDNA is a structural component in the A. pleuropneumoniae - P. multocida biofilm but not for the other biofilms. With respect to proteins, an increase in staining was observed in the biofilms of A. pleuropneumoniae - P. multocida. However, the biofilm was resistant to proteinase $\mathrm{K}$ treatment as observed for the other multi-species biofilms.

Concluding, the analysis of the biofilm matrix indicates that PGA remains a major component responsible for the integrity of the biofilm. However, eDNA also play a role in the integrity of the A. pleuropneumoniae - $P$. multocida biofilm.

\section{Confirmation of the presence of $A$. pleuropneumoniae in multi-species biofilms by FISH}

To confirm the presence of $A$. pleuropneumoniae in these multi-species biofilms, FISH assays were performed for the A. pleuropneumoniae-S suis and A. pleuropneumoniae - $B$. bronchiseptica biofilms. In both cases, the presence of $A$. pleuropneumoniae was confirmed in the biofilms (Fig. 4). However, its distribution in the biofilms resulted different. For the A. pleuropneumoniae-S. suis biofilm, $A$. pleuropneumoniae appears in a layer on top of the biofilm and $S$. suis in the bottom of the same (Fig. 4 and 5a). For the A. pleuropneumoniae-B. bronchiseptica biofilm, $A$. pleuropneumoniae was mainly observed at the bottom of the biofilm whereas $B$. bronchiseptica was mainly on top of the biofilm (Fig. 4 and $5 b)$.

\section{Discussion}

This study demonstrates that A. pleuropneumoniae is able to form multi-species biofilms with other bacteria isolated from the swine respiratory tract. Importantly, $A$. pleuropneumoniae was able to form strong biofilms with other respiratory pathogen of swine belonging to PRDC (S. suis, B. bronchiseptica and P. multocida), and with a nasal isolate of $S$. aureus whereas $A$. pleuropneumoniae biofilm formation was decrease in the presence of the intestinal pathogen, E. coli. Furthermore, the key finding was that $A$. pleuropneumoniae could satisfy its requirements of pyridine compounds from other swine pathogens to grow and form biofilms. The fact that $A$. pleuropneumoniae was able to get pyridine compounds (probably NAD, NMN or NR [30, 31]) from other bacteria suggest that $A$. pleuropneumoniae could be using this strategy to form multi-species biofilms and persist in its host $[8,17]$ and/or to survive in the environment $[7,32]$.

Knowledge regarding the ability of swine respiratory pathogens, such as A. pleuropneumoniae, to form multispecies biofilms is limited and could provide important clues for processes during infection and persistence in the host and in the environment [7, 9]. Respiratory diseases in pigs have a polymicrobial nature [11, 33], but it is not yet established if porcine respiratory diseases could involve multi-species biofilms. However, it has been reported that several human and animal pathogens, such as Pseudomonas aeruginosa, Stenotrophomonas maltophilia, Legionella pneumophila, Klebsiella pneumoniae, Fusobacterium nucleatum, Streptococcus mutans and $E$. coli, can form multi-species biofilms, and these biofilms help them to increase their pathogenicity, 


\begin{tabular}{|c|c|c|c|c|c|c|}
\hline \multirow[b]{2}{*}{ App 719 NAD + } & $\begin{array}{l}\text { FM 1-43 } \\
\text { (cells) }\end{array}$ & $\begin{array}{l}\text { Syto } 9 \\
\text { (live cells) }\end{array}$ & $\begin{array}{l}\text { Propidium iodide } \\
\text { (dead cells) }\end{array}$ & $\begin{array}{l}\text { WGA } \\
\text { (PGA) }\end{array}$ & \multirow[t]{2}{*}{$\begin{array}{l}\text { BOBO } \\
\text { (eDNA) }\end{array}$} & \multirow[t]{2}{*}{$\begin{array}{l}\text { Sypro Ruby } \\
\text { (Proteins) }\end{array}$} \\
\hline & & & & & & \\
\hline App 719 NAD - & & & & & & \\
\hline App - Ss NAD - & & & & & & \\
\hline $\mathrm{App}$ - Bb NAD - & & & & & so & \\
\hline App - Pm NAD - & & & & & & \\
\hline App - Sa NAD - & & & & & & \\
\hline App - Ec NAD - & & & & & & \\
\hline $\mathrm{App}$ - Ss - Bb NAD - & & & & & & \\
\hline App - Ss - Ec NAD - & & & & & & \\
\hline $\mathrm{App}-\mathrm{Bb}-\mathrm{Ec} \mathrm{NAD}-$ & & & & & & \\
\hline $\begin{array}{l}\text { Fig. } 3 \text { CLSM of multi-sp } \\
\text { suis, B. bronchiseptica, } P \text {. } \\
1-43, \text { SYTO 9, propidium } \\
\text { poly-N-acetylglucosamir } \\
\text { coli. Scale bar } 30 \mu \mathrm{m}\end{array}$ & 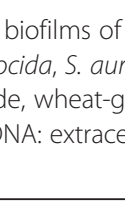 & eumonic & $\begin{array}{l}\text { other swine pathe } \\
\text { ingle, dual or triple } \\
\text { gon green, BOBO- } \\
\text { oneumoniae; Ss: S. }\end{array}$ & & $\begin{array}{l}\text { I from Invitrog } \\
\mathrm{c} a \text {; Pm: P. mult }\end{array}$ & $\begin{array}{l}\text { ropneumoniae, } \\
\text { stained with F } \\
\text { ne, OR). PGA: } \\
\text { : S. aureus; Ec: }\end{array}$ \\
\hline
\end{tabular}

resistance and their persistence in the environment [22, 24-26, 34-41]. The lower respiratory tract, has a limited essential nutrients supply for bacterial growth [8]. Although mammalian cells contain significant amounts of $\operatorname{NAD}(\mathrm{P})(\mathrm{H})$, the supply of pyridine nucleotides in extracellular fluids is probably quite low because several types of mammalian cells possess extrinsic $\mathrm{NAD}(\mathrm{P})+$ nucleosidases [30]. In response of this, A. pleuropneumoniae has developed a number of virulence mechanisms to overcome this lack, such as cell lysis that allows the release of nutrients into the surrounding environment [8]. Beside, multi-species biofilm formation by this bacterium with other porcine respiratory pathogens, such as $S$. suis, B. bronchiseptica and P. multocida; and other isolated bacteria from the porcine respiratory tract, among which $S$. aureus could be important either for chronic infections development or persistence, where A. pleuropneumoniae could be obtained these pyridine compounds 


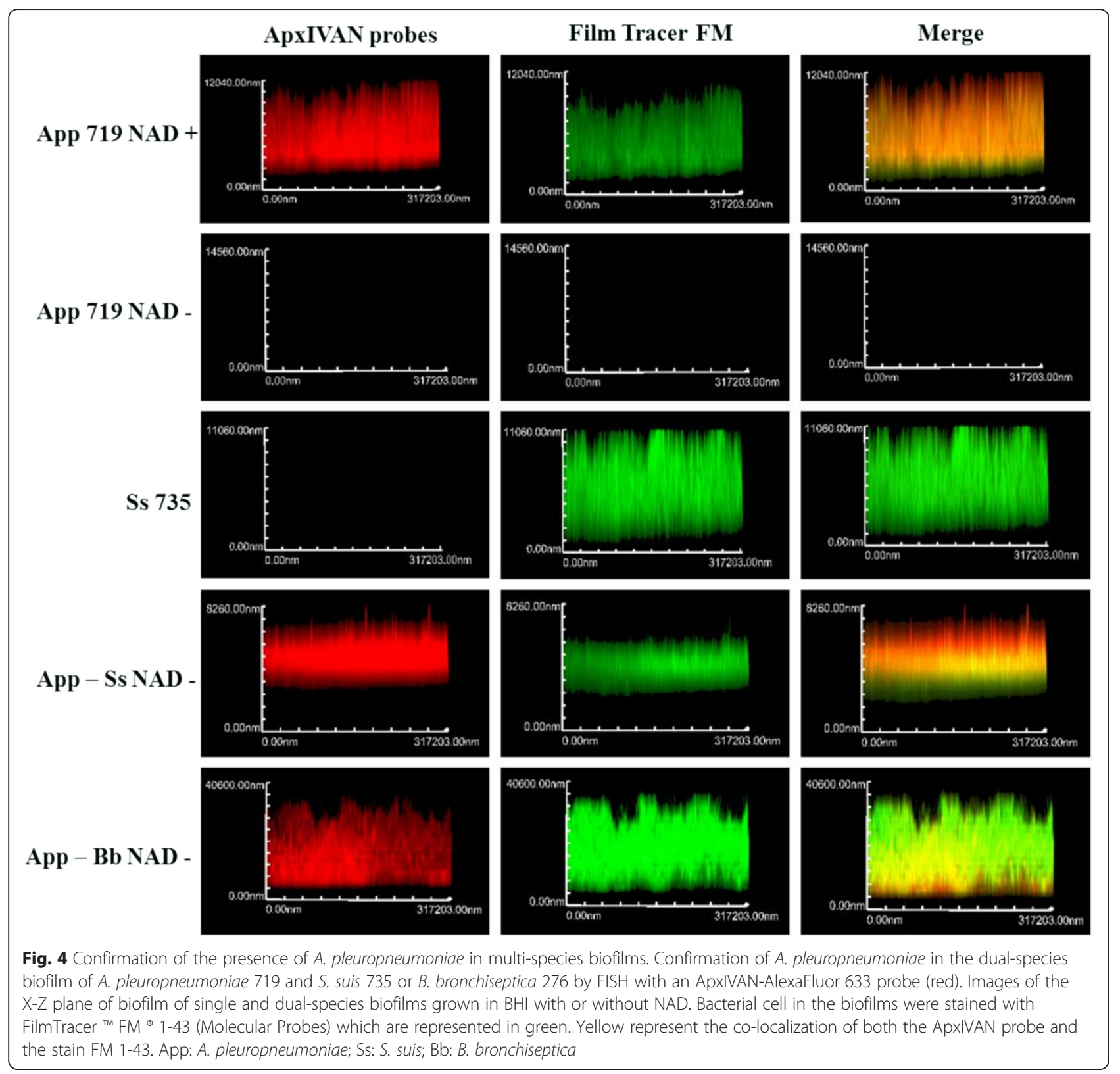

from the other bacteria without causing further host damage; as described in several other diseases [42, 43]. Furthermore, multi-species biofilm formation could be allowing that $A$. pleuropneumoniae survive outside the host in the environment [7,32]. More studies are needed to know the exact role of these multi-species communities and if it could play of role in the case of PRDC.

In $A$. pleuropneumoniae, biofilms formation over polystyrene microtiter plates depends on PGA production [9, $18,27,44]$ but protein and eDNA have also been reported [17]. PGA is the main component responsible for the multi-species biofilms integrity. However, some biofilms were less sensitive to dispersin B treatment suggesting that other matrix components are required for the multi-species biofilms integrity. Moreover, knowledge is limited regarding changes in the extracellular matrix composition when single-species biofilms become multi-species $[45,46]$. Here, variations in the PGA distribution were observed in most multi-species biofilms with the presence of clusters or filament-like structures. Changes in the polysaccharide distribution could be related with the formation of small clusters by A. pleuropneumoniae, or by the production of additional polysaccharides by the secondary species, as E. coli, which produces cellulose to form biofilms [9]. Different polysaccharides production is supported by the fact that E. coli multi-species biofilms were resistant to the treatments with dispersin B, DNase I and proteinase K. 

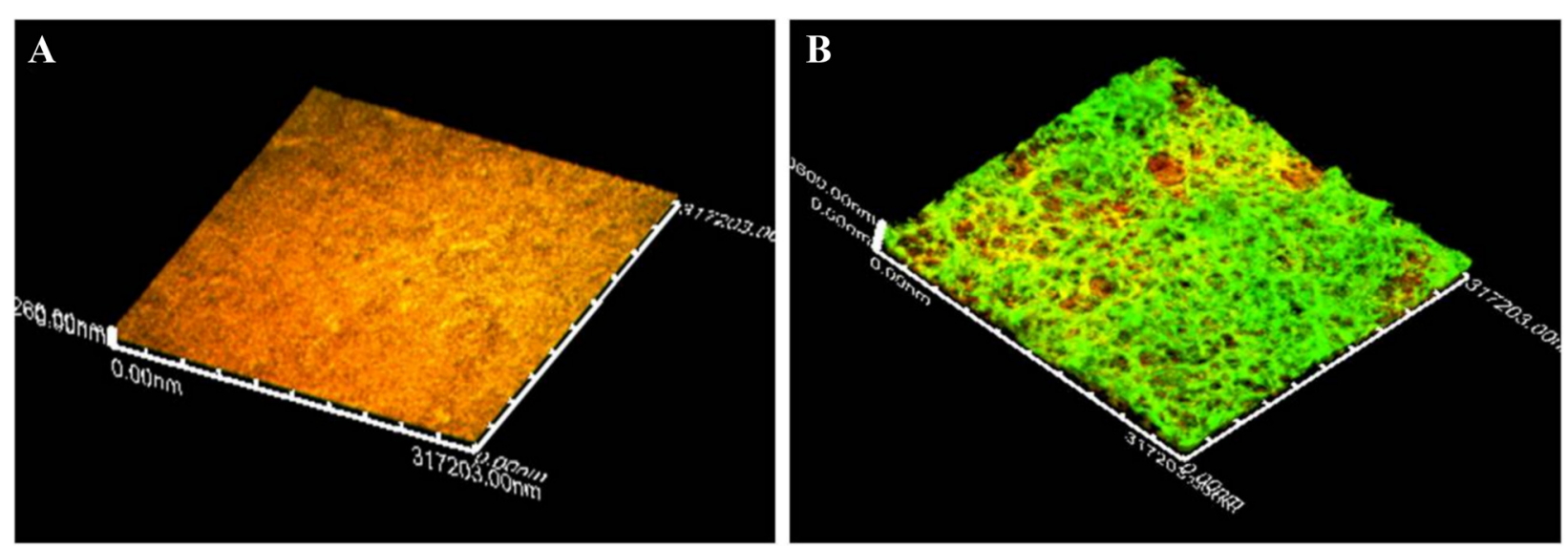

Fig. 53 D models of A. pleuropneumoniae in multi-species biofilms. Duals-species biofilms of (a) A. pleuropneumoniae 719 and S. suis 735 or (b) A. pleuropneumoniae 719 and $B$. bronchiseptica 276 detected with ApxIVAN-AlexaFluor 633 probe (red). Bacterial cell in the biofilms were stained with FilmTracer ${ }^{\mathrm{TM}} \mathrm{FM}^{\circledR}{ }^{\circledR 1-43}$ (Molecular Probes) which are represented in green. Yellow represent the co-localization of both the ApxIVAN probe and FM 1-43

Interestingly, eDNA an important factor in multispecies biofilms $[47,48]$ was identified as a matrix component for the A. pleuropneumoniae - P. multocida dual-species when NAD was not supplemented. There was important increase in the eDNA staining, and the biofilm were sensitive to DNase I treatment. This was not observed for A. pleuropneumoniae single-species biofilms. Hathroubi et al., [49] recently report changes in the structure composition of the A. pleuropneumoniae extracellular matrix when exposed to sub-inhibitory concentrations (sub-MIC) of penicillin G, noting that eDNA play a structural role, they concluded that because subMIC of penicillin G induce cell death in a limited population, this allows the release of chromosomal DNA, which in turn could be used by the surviving bacteria to build the biofilm. Here, was observed a limited number of dead bacteria This change was only observed in the biofilm formed by $A$. pleuropneumoniae with $P$. multocida without NAD supplementation. More studies are necessaries to understand the importance of eDNA in multi-species biofilms.

Additionally, Disperin B was unable to completely segregate the multi-species biofilm formed by both bacteria. Indicating that $P$. multocida is actively participating in the structure of the extracellular matrix components. Unfortunately, little information exists about the ability of $P$. multocida to form biofilms and its extracellular matrix composition [50-52]. This highlights that fact multi-species biofilm composition depends directly on the species involved in the biofilm formation.

\section{Conclusion}

In conclusion, our data show that $A$. pleuropneumoniae has the ability to form multi-species biofilms with respiratory porcine pathogens, S. suis, B. bronchiseptica, and P. multocida, and with other bacteria isolated from pigs. Furthermore, we report for the first time that $A$. pleuropneumoniae is able to satisfy the requirement of pyridine compounds through of other bacteria and this supported its growth and biofilm formation. Further research is needed to understand the pathway by which $A$. pleuropneumoniae can obtain its requirements of pyridine compounds, besides if these multi-species biofilm interactions are involved in the onset and/or development of swine respiratory diseases. Furthermore, the multi-species biofilm interactions could increase the resistance and/or persistence of A. pleuropneumoniae in its host, and in the environment.

\section{Additional file}

Additional file 1: Figure $\mathbf{S 1}$. CLSM of multi-species biofilms of $A$. pleuropneumoniae with the other swine pathogens with NAD supplementation. A. pleuropneumoniae, S. suis, B. bronchiseptica, P. multocida, S. aureus and $E$. coli grown in single, dual or triple-species biofilms in $\mathrm{BH}$ media with NAD stained with FM 1-43, SYTO 9, propidium iodide, wheat-germ agglutinin (WGA)-Oregon green, BOBO-3, and SYPRO Ruby (all from Invitrogen, Eugene, OR). PGA: poly-N-acetylglucosamine; eDNA: extracellular DNA; App: A. pleuropneumoniae; Ss: S. suis; Bb: B. bronchiseptica; Pm: P. multocida; Sa: S. aureus; Ec: E. coli. Scale bar 30 m (TIF 37984 kb)

\section{Abbreviations}

NMN, nicotinamide mononucleotide; NR, nicotinamide riboside; NAD, nicotinamide adenine dinucleotide; $\mathrm{BHI}$, brain heart infusion agar; $\mathrm{CFU}$, colony forming units; PGA, N-acetyl-D-glucosamine; FISH, Fluorescent in situ hybridization

\section{Acknowledgements}

This project was supported by a grant from CONACYT, Mexico (No. 258863) and Discovery Grant (003428 to MJ) from the Natural Sciences and Engineering Research Council of Canada (NSERC). The authors would also like to acknowledge Frédéric Berthiaume for assistance with the confocal microscopy and Profs. M. Archambault, J.M. Fairbrother and M. Gottschalk for bacterial strains. 


\section{Funding}

This project was supported by a grant from CONACYT, Mexico (No. 258863) and Discovery Grant (003428 to MJ) from the Natural Sciences and Engineering Research Council of Canada (NSERC).

\section{Authors' contributions}

Conceived and designed the experiments: ALM, YDNT, JL, MJ, ALGB; Performed the experiments: ALM; Analyzed the data: ALM, YDNT, JL, MJ, FJAG, ALGB; Wrote the paper: ALM, YDNT, MJ, FJAG, RON, ALGB. All authors read and approved the final manuscript.

\section{Competing interests}

The authors declare that they have no competing interests.

\section{Ethics and consent to participate}

No formal ethics approval was required in this particular case.

\begin{abstract}
Author details
${ }^{1}$ Centro de Ciencias Básicas, Universidad Autónoma de Aguascalientes, Aguascalientes, Ags., Mexico20131. ${ }^{2}$ Groupe de recherche sur la maladies infectieuses du porc, Faculté de médecine vétérinaire, Université de Montréal, St-Hyacinthe, Québec J2S 7C6, Canada. ${ }^{3}$ Departamento de Microbiología Molecular, Instituto de Biotecnología, Universidad Nacional Autónoma de México, Cuernavaca, Morelos, Mexico62260. ${ }^{4}$ Laboratorio de Biología Celular y Tisular, Departamento de Morfología, Centro de Ciencias Básicas, Universidad Autónoma de Aguascalientes, Aguascalientes, Ags. Mexico20131.
\end{abstract}

Received: 24 June 2015 Accepted: 15 June 2016 Published online: 27 June 2016

\section{References}

1. Brockmeier S, Halbur P, Thacker, E. Porcine Respiratory Disease Complex. In: Brogden K, Guthmiller J, editors. Polymicrobial Diseases. ASM Press: American Society for Microbiology; 2002;231-58.

2. Ramjeet M, Cox A, Hancock M, Mourez M, Labrie J, et al. Mutation in the LPS outer core biosynthesis gene, galU, affects LPS interaction with the RTX toxins Apxl and Apxll and cytolytic activity of Actinobacillus pleuropneumoniae serovar 1. Mol Microbiol. 2008;70:221-35.

3. Auger E, Deslandes V, Ramjeet M, Contreras I, Nash J, et al. Host Pathogen Interactions of Actinobacillus pleuropneumoniae with Porcine Lung and Tracheal Epithelial Cells. Infect Immun. 2009;77:1426-41.

4. Buettner F, Konze S, Maas A, Gerlach G. Proteomic and immunoproteomic characterization of a DIVA subunit vaccine against Actinobacillus pleuropneumoniae. Proteome Sci. 2011:9:1-23.

5. Li L, Xu Z, Zhou Y, Li T, Sun L, et al. Analysis on Actinobacillus pleuropneumoniae LuxS regulated genes reveals pleiotropic roles of LuxS/AI2 on biofilm formation, adhesion ability and iron metabolism. Microb Pathog. 2011;50:293-302.

6. Li L, Xu Z, Zhou Y, Sun L, Liu Z, et al. Global Effects of Catecholamines on Actinobacillus pleuropneumoniae Gene Expression. PLoS ONE. 2012;7:e31121.

7. Loera-Muro V, Jacques M, Tremblay Y, Avelar-González F, Loera-Muro A, et al. Detection of Actinobacillus pleuropneumoniae in drinking water from pig farms. Microbiology. 2013;159:535-43.

8. Chiers K, De Waele T, Pasmans F, Ducatelle R, Haesebrouck F. Virulence factors of Actinobacillus pleuropneumoniae involved in colonization, persistence and induction of lesions in its porcine host. Vet Res. 2010;41:65-70.

9. Jacques M, Aragon V, Tremblay YD. Biofilm formation in bacterial pathogens of veterinary importance. Anim Health Res Rev. 2010;11:97-121.

10. Klitgaard K, Friis C, Jensen T, Angen $\varnothing$, Boye M. Transcriptional Portrait of Actinobacillus pleuropneumoniae during Acute Disease - Potential Strategies for Survival and Persistence in the Host. PLOS ONE. 2012;7:e35549. doi:10. 1371/journal.pone.0035549.

11. Loera-Muro A, Avelar-González F, Loera-Muro V, Jacques M, Guerrero-Barrera A. Presence of Actinobacillus pleuropneumoniae, Streptococcus suis, Pasteurella multocida, Bordetella bronchiseptica, Haemophilus parasuis and Mycoplasma hyopneumoniae in upper respiratory tract of swine in farms from Aguascalientes. Mexico OJAS. 2013:3:132-7.

12. Perry MB, Angen $\mathrm{O}$, Maclean $\mathrm{LL}$, Lacouture $\mathrm{S}$, Kokotovic $B$, et al. An atypica biotype I Actinobacillus pleuropneumoniae serovar 13 is present in North America. Vet Microbiol. 2011;4:403-10.
13. Lone A, Deslandes V, Nash J, Jacques M, Maclnnes J. malT knockout mutation invokes a stringent type gene-expression profile in Actinobacillus pleuropneumoniae in bronchoalveolar fluid. BMC Microbiol. 2009; doi: 10.1186/1471-2180-9-195

14. Stevenson A, Macdonald J, Roberts M. Cloning and characterization of type 4 fimbrial genes from Actinobacillus pleuropneumoniae. Vet Microbiol. 2003; 92:121-34.

15. Boekema BK, Van Putten JP, Stockhofe-Zurwieden N, Smith HE. Host cell contact-induced transcription of the type IV fimbria gene cluster of Actinobacillus pleuropneumoniae. Infect Immun. 2004;72:691-700.

16. Labrie J, Pelletier-Jacques G, Deslandes V, Ramjjet M, Nash J, et al. Effects of growth conditions on biofilm formation by Actinobacillus pleuropneumoniae. Vet Res. 2010:41:3-10.

17. Wu C, Labrie J, Tremblay YD, Haine D, Mourez M, et al. Zinc as an agent for the prevention of biofilm formation by pathogenic bacteria. J Appl Microbiol. 2013;115:30-40.

18. Bello-Ortí B, Deslandes V, Tremblay YD, Labrie J, Howell KJ, Tucker AW, Maskell DJ, Aragon V, Jacques M. Biofilm formation by virulent and non-virulent strains of Haemophilus parasuis. Vet Res. 2015;45:104.

19. Costerton JW, Stewart PS, Greenberg EP. Bacterial biofilms: a common cause of persistent infections. Science. 1999;284:1318-22.

20. Stoodley P, Sauer K, Davies DG, Costerton JW. Biofilms as complex differentiated communities. Annu Rev Microbiol. 2002:56:187-209.

21. Bjarnsholt T, Alhede M, Alhede M, Eickhardt-Sørensen SR, Moser C, et al. The in vivo biofilm. Trends Microbiol. 2013;21:466-74.

22. Yang L, Liu Y, Wu H, Høiby N, Molin S, et al. Current understanding of multispecies biofilms. Int J Oral Sci. 2011;3:74-81.

23. Fröls S. Archaeal biofilms: widespread and complex. Biochem Soc Trans. 2013:41:393-8

24. Orell A, Fröls S, Albers SV. Archaeal Biofilms: The Great Unexplored. Annu Rev Microbiol. 2013;67:337-54.

25. Serra DO, Richter AM, Klauck G, Mika F, Hengge R. Microanatomy at cellular resolution and spatial order of physiological differentiation in a bacterial biofilm. mBio. 2013; doi: 10.1128/mBio.00103-13

26. Standar K, Kreikemeyer B, Redanz S, Münter WL, Laue M, Podbielski A. Setup of an In Vitro Test System for Basic Studies on Biofilm Behavior of Mixed- Species Cultures with Dental and Periodontal Pathogens. PLoS ONE. 2010; doi: 10. 1371/journal.pone.0013135

27. Tremblay YD, Lamarche D, Chever P, Haine D, Messier S, et al. Characterization of the ability of coagulase-negative staphylococci isolated from the milk of Canadian farms to form biofilms. J Dairy Sci. 2013;96:234-46.

28. Jensen HE, Gyllensten J, Hofman C, Leifsson PS, Agerholm JS, et al. Histologic and bacteriologic findings in valvular endocarditis of slaughter-age pigs. J Vet Diagn Invest. 2010;22:921-7.

29. Wongnarkpet S, Pfeiffer DU, Morris RS, Fenwick SG. An on-farm study of the epidemiology of Actinobacillus pleuropneumoniae infection in pigs as part of a vaccine efficacy trial. Prev Vet Med. 1998;12:1-11.

30. O'Reilly T, Niven DF. Defining the Metabolic and Growth Responses of Porcine Haemophili to Exogenous Pyridine Nucleotides and Precursors. J Gen Microbiol. 1986;132:807-18

31. Martin PR, Shea RJ, Mulks MH. Identification of a Plasmid-Encoded Gene from Haemophilus ducreyi Which Confers NAD Independence. J Bacteriol. 2001:183:1168-74

32. Assavacheep P, Rycroft AN. Survival of Actinobacillus pleuropneumoniae outside the pig. Res Vet Sci. 2012;94:22-6.

33. Opriessnig T, Giménez-Lirola LG, Halbur PG. Polymicrobial respiratory disease in pigs. Anim Health Res Rev. 2011;12:133-48.

34. Biyikoglu B, Ricker A, Diaz PI. Strain-specific colonization patterns and serum modulation of multi-species oral biofilm development. Anaerobe. 2012;18:459-70.

35. Bridier A, Sanchez-Vizuete MP, Le Coq D, Aymerich S, Meylheuc T, et al. Biofilms of a Bacillus subtilis Hospital Isolate Protect Staphylococcus aureus from Biocide Action. PLoS ONE. 2012;7:e44506. doi:10.1371/journal.pone.0044506.

36. de Chávez Paz LE. Development of a Multispecies Biofilm Community by Four Root Canal Bacteria. J Endod. 2012:38:318-23.

37. Lopes SP, Ceri H, Azevedo NF, Pereira MO. Antibiotic resistance of mixed biofilms in cystic fibrosis: impact of emerging microorganisms on treatment of infection. Int J Antimicrob Agents. 2012;40:260-3.

38. Stewart CR, Muthye V, Cianciotto NP. Legionella pneumophila Persists within Biofilms Formed by Klebsiella pneumoniae, Flavobacterium sp., and Pseudomonas fluorescens under Dynamic Flow Conditions. PLoS ONE. 2012; doi: 10.1371/journal.pone.0050560. 
39. Ryan RP, Fouhy Y, Garcia BF, Watt S, Niehaus K, et al. Interspecies signalling via the Stenotrophomonas maltophilia diffusible signal factor influences biofilm formation and polymyxin tolerance in Pseudomonas aeruginosa. Mol Microbiol. 2008:68:75-86.

40. Almeida C, Azevedo N, Santos S, Keevil C, Vieira M. Discriminating Multispecies Populations in Biofilms with Peptide Nucleic Acid Fluorescence In Situ Hybridization (PNA FISH). PLoS ONE. 2011;6:e14786. doi:10.1371/ journal.pone.0014786.

41. Schlafer S, Raarup MK, Wejse PL, Nyvad B, Städler BM, et al. Osteopontin Reduces Biofilm Formation in a Multi-Species Model of Dental Biofilm. PLoS ONE. 2012;7:e41534. doi:10.1371/journal.pone.0041534.

42. Burmølle M, Thomsen TR, Fazli M, Dige I, Christensen L, Homøe P, Tvede M, Nyvad B, Tolker-Nielsen T, Givskov M, Moser C, Kirketerp-Møller K, Johansen HK, Høiby N, Jensen PØ, Sørensen SJ, Bjarnsholt T. Biofilms in chronic infections -a matter of opportunitymonospecies biofilms in multispecies infections. FEMS Immunol Med Microbiol. 2010;59:324-36. doi:10.1111/j.1574-695X.2010.00714.x.

43. Bertesteanu S, Triaridis S, Stankovic M, Lazar V, Chifiriuc MC, Vlad M, Grigore R. Polymicrobial wound infections: Pathophysiology and current therapeutic approaches. Int J Pharm. 2014;463:119-26. doi:10.1016/j.jpharm.2013.12.012.

44. Kaplan JB, Velliyagounder K, Ragunath C, Rohde H, Mack D, et al. Genes involved in the synthesis and degradation of matrix polysaccharide in Actinobacillus actinomycetemcomitans and Actinobacillus pleuropneumoniae biofilms. J Bacteriol. 2004;186:8213-20.

45. Ali Mohammed MM, Nerland AH, Al-Haroni M, Bakken V. Characterization of extracellular polymeric matrix, and treatment of Fusobacterium nucleatum and Porphyromonas gingivalis biofilms with DNase I and proteinase K. J Oral Microbiol. 2013; doi: 10.3402/jom.v5i0.20015

46. Dominiak D, Nielsen JN, Nielsen PH. Extracellular DNA is abundant and important for microcolony strength in mixed microbial biofilms. Environ Microbiol. 2011;13:710-21.

47. Jakubovics NS, Shields RC, Rajarajan N, Burgess JG. Life after Death: The Critical Role of Extracellular DNA in Microbial Biofilms. Lett Appl Microbiol. 2013;57:467-75.

48. Tang L, Schramm A, Neu T, Revsbech NP, Meyer RL. Extracellular DNA in adhesion and biofilm formation of four environmental isolates: a quantitative study. FEMS Microbiol Ecol. 2013:86:394-403.

49. Hathroubi S, Fontaine-Gosselin SE, Tremblay YDN, Labrie J, Jacques M Sub-inhibitory concentrations of penicillin $\mathrm{G}$ induce biofilm formation by field isolates of Actinobacillus pleuropneumoniae. Vet Microbiol. 2015; 179(3-4):277-86. doi:10.1016/j.vetmic.2015.06.011.

50. Olson ME, Ceri H, Morck DW, Buret AG, Read RR. Biofilm bacteria: formation and comparative susceptibility to antibiotics. Can J Vet Res. 2002;66:86-92.

51. Rajagopal R, Nair GK, Mini M, Joseph L, Saseendranath MR, John K. Biofilm formation of Pasteurella multocida on bentonite clay. Iran J Microbiol. 2013;5:120-5.

52. Romanò CL, De Vecchi E, Vassena C, Manzi G, Drago L. A case of a late and atypical knee prosthetic infection by no-biofilm producer Pasteurella multocida strain identified by pyrosequencing. Pol J Microbiol. 2013;62:435-8.

\section{Submit your next manuscript to BioMed Central and we will help you at every step:}

- We accept pre-submission inquiries

- Our selector tool helps you to find the most relevant journal

- We provide round the clock customer support

- Convenient online submission

- Thorough peer review

- Inclusion in PubMed and all major indexing services

- Maximum visibility for your research

Submit your manuscript at www.biomedcentral.com/submit

) Biomed Central 